Pacific

Journal of

Mathematics

ON A QUESTION OF A. BALOG

ILYA D. SHKREDOV 


\title{
ON A QUESTION OF A. BALOG
}

\author{
ILYA D. SHKREDOV
}

We give a partial answer to a conjecture of $A$. Balog concerning the size of $A A+A$, where $A$ is a finite subset of real numbers. We also prove several new results on the cardinality of $A: A+A, A A+A A$ and $A: A+A: A$.

\section{Introduction}

Let $A \subset \mathbb{R}$ be a finite set. Define the sumset, and respectively the product set, by

$$
A+A:=\{a+b: a, b \in A\}
$$

and

$$
A A:=\{a b: a, b \in A\} .
$$

The Erdős-Szemerédi conjecture [1983] states that for all $\varepsilon>0$,

$$
\max \{|A+A|,|A A|\} \gg|A|^{2-\varepsilon} .
$$

Loosely speaking, the conjecture says that any set of reals (or integers) cannot be highly structured in both a multiplicative and an additive sense. The best result in this direction is due to Solymosi [2009].

Theorem 1. Let $A \subset \mathbb{R}$ be a set. Then

$$
\max \{|A+A|,|A A|\} \gg|A|^{4 / 3} \log ^{-1 / 3}|A| .
$$

If one considers the set

$$
A A+A=\{a b+c: a, b, c \in A\}
$$

then the Erdôs-Szemerédi conjecture implies that $A A+A$ has size at least $|A|^{2-\varepsilon}$ (we assume for simplicity that $1 \in A$ ). Balog [2011] formulated the weaker hypothesis that for all $\varepsilon>0$ one has

$$
|A A+A| \gg|A|^{2-\varepsilon} .
$$

In that paper he proved the following result, which implies, in particular, that $|A A+A| \gg|A|^{3 / 2}$ and $|A A+A A| \gg|A||A: A|^{1 / 2}$.

This work was supported by Russian Scientific Foundation grant 14-11-00433.

MSC2010: 11B13, 11B75.

Keywords: sum-products, Szemerédi-Trotter theorem. 
Theorem 2. For every finite set of reals $A, B, C, D \subset \mathbb{R}$, we have

$$
|A C+A||B C+B| \gg|A||B||C|
$$

and

$$
|A C+A D||B C+B D| \gg|B: A||C||D| .
$$

More precisely (see [Schoen and Shkredov 2013]),

$$
|(A \times B) \cdot \Delta(C)+A \times B| \gg|A||B||C|
$$

and

$$
|(A \times B) \cdot \Delta(C)+(A \times B) \cdot \Delta(D)| \gg|B: A||C||D|,
$$

where

$$
\Delta(A):=\{(a, a): a \in A\} .
$$

Murphy et al. [2015] obtained a partial answer to a "dual" question on the size of $A(A+A)$. The main result of this paper is the following new bound for $A: A+A$ and $A A+A$, stated more precisely in Theorem 12 .

Theorem 3. Let $A$ be a finite subset of positive reals. Then there is $\varepsilon_{1}>0$ such that

$$
|A: A+A| \gg|A|^{3 / 2+\varepsilon_{1}} .
$$

Moreover, if $|A: A| \ll|A A|$ then there exists $\varepsilon_{2}>0$ such that

$$
|A A+A| \gg|A|^{3 / 2+\varepsilon_{2}} .
$$

We also prove several results on the cardinality of $A A+A A$ and $A: A+A: A$; see Theorem 14 and Proposition 15 below.

Roche-Newton and Zhelezov [2015] conjectured there exist absolute constants $c$ and $c^{\prime}$ such that for any finite $A \subset \mathbb{C}$,

$$
\left|\frac{A+A}{A+A}\right| \leq c|A|^{2} \Longrightarrow|A+A| \leq c^{\prime}|A| .
$$

Similar conjectures were made for the sets $(A-A) /(A-A),(A-A)(A-A)$, $A(A+A+A+A)$ and so on. We conclude this paper by giving a partial answer to a variant of Roche-Newton and Zhelezov's conjecture:

$$
|(A+A)(A+A)+(A+A)(A+A)| \ll|A|^{2} \Longrightarrow|A \pm A| \ll|A| \log |A| ;
$$

see Corollary 17.

The main idea of the proof of Theorem 3 is the following. We need to estimate from below the sumset of two sets $A$ and $A: A$. As in many problems of this type, the usual applications of the Szemerédi-Trotter theorem [Tao and Vu 2006] or Solymosi's method [Balog 2011] give us a lower bound of the form $|A: A+A| \gg|A|^{3 / 2}$. In [Schoen and Shkredov 2011] the exponent $3 / 2$ was improved in the particular 
case of sumsets of convex sets. After that the method was developed by several authors; see, e.g., [Konyagin and Rudnev 2013; Li 2011; Li and Roche-Newton 2012; Schoen 2014; Schoen and Shkredov 2013; Shkredov 2013a; 2013b; 2015]. In [Shkredov 2015] it was proved that the bound $|A+B| \gg|A|^{3 / 2+c}, c>0$, holds for a wide class of different sets having roughly comparable sizes. For example, such a bound holds if $A$ and $B$ have small multiplicative doubling. It turns out that if (3) cannot be improved then there is some large set $C$ such that $|A C| \ll|A|$. This allows us to apply results from [Shkredov 2015].

\section{Notation}

Let $\boldsymbol{G}$ be an abelian group and + be the group operation. We use the same letter to denote a set $S \subseteq \boldsymbol{G}$ and its characteristic function $S: \boldsymbol{G} \rightarrow\{0,1\}$. By $|S|$ denote the cardinality of $S$.

Let $f, g: \boldsymbol{G} \rightarrow \mathbb{C}$ be two functions with finite supports. Put

$$
(f * g)(x):=\sum_{y \in \boldsymbol{G}} f(y) g(x-y) \quad \text { and } \quad(f \circ g)(x):=\sum_{y \in \boldsymbol{G}} f(y) g(y+x) .
$$

Let $A \subseteq \boldsymbol{G}$ be a set. For any real $\alpha>0$ let

$$
\mathrm{E}_{\alpha}^{+}(A)=\sum_{x \in \boldsymbol{G}}(A \circ A)^{\alpha}(x)
$$

be the higher energy of $A$. In the particular case $\alpha=2$ we write $\mathrm{E}^{+}(A)=\mathrm{E}_{2}^{+}(A)$ and $\mathrm{E}(A, B)$ for $\sum_{x \in G}(A \circ A)(x)(B \circ B)(x)$. The quantity $\mathrm{E}^{+}(A)$ is called the additive energy of a set; see, e.g., [Tao and Vu 2006]. For a sequence $s=\left(s_{1}, \ldots, s_{k-1}\right)$ put $A_{s}^{+}=A \cap\left(A-s_{1}\right) \cap \cdots \cap\left(A-s_{k-1}\right)$. Then

$$
\mathrm{E}_{k}^{+}(A)=\sum_{s_{1}, \ldots, s_{k-1} \in \boldsymbol{G}}\left|A_{s}^{+}\right|^{2} .
$$

If we have a group $G$ with multiplication instead of addition, then we use the symbol $\mathrm{E}_{\alpha}^{\times}(A)$ for the corresponding energy of a set $A$ and we write $A_{s}^{\times}$for $A \cap\left(A s_{1}^{-1}\right) \cap \cdots \cap\left(A s_{k-1}^{-1}\right)$. In the case of a unique operation we write just $\mathrm{E}_{k}(A)$, $\mathrm{E}(A)$ and $A_{s}$.

Let $A, B \subseteq \boldsymbol{G}$ be two finite sets. The magnification ratio $R_{B}[A]$ of the pair $(A, B)$ (see, e.g., [Tao and Vu 2006]) is defined by

$$
R_{B}[A]=\min _{\varnothing \neq Z \subseteq A} \frac{|B+Z|}{|Z|} .
$$

A beautiful result on the magnification ratio was proven by Petridis [2012]. 
Theorem 4. For any $A, B, C \subseteq \boldsymbol{G}$, we have

$$
|B+C+X| \leq R_{B}[A] \cdot|C+X|,
$$

where $X \subseteq A$ and $|B+X|=R_{B}[A]|X|$.

We conclude the section with Ruzsa's triangle inequality; see, e.g., [Tao and Vu 2006]. Interestingly, our proof (developing some ideas of [Schoen and Shkredov 2013; Murphy et al. 2015]) describes the situation when the triangle inequality is sharp, namely, when $|B \cap(A-z)-C| \approx|C|$ for many $z \in A-B$.

Lemma 5. Let $A, B, C \subseteq \boldsymbol{G}$ be any sets. Then

$$
|C||A-B| \leq|A \times B-\Delta(C)| \leq|A-C||B-C| .
$$

Proof. We have

$$
|A \times B-\Delta(C)|=\sum_{z \in A-B}|B \cap(A-z)-C| \geq|A-B||C| .
$$

The inequality above is trivial and the identity follows by the projection of points $(x, y) \in A \times B-\Delta(C),(x, y)=(a-c, b-c), a \in A, b \in B, c \in C$, onto $z:=x-y=a-b \in A-B$. If $z$ is fixed we see that the result of the projection is the intersection of the line $z=x-y$ with our set and moreover the ordinates of the points from the intersection belong to $B \cap(A-z)-C$. It is easy to check that the converse is also true.

All logarithms are base 2 . The signs $\ll$ and $\gg$ are the usual Vinogradov symbols.

\section{Preliminaries}

As we discussed in the introduction our proof uses some notions from [Shkredov 2015]. So, let us recall the main definition of that paper.

Definition 6. A set $A \subset \boldsymbol{G}$ has SzT-type (in other words, $A$ is called a SzemerédiTrotter set) with parameter $\alpha \geq 1$ if for any set $B \subset \boldsymbol{G}$ and an arbitrary $\tau \geq 1$,

$$
|\{x \in A+B:(A * B)(x) \geq \tau\}| \ll c(A)|B|^{\alpha} \cdot \tau^{-3},
$$

where $c(A)>0$ is a constant that depends on the set $A$ only.

Simple calculations (or see [Shkredov 2015, Lemma 7]) give us some connections between various energies of SzT-type sets. Formula (11) below is due to Li [2011].

Lemma 7. Suppose that $A, B, C \subseteq \boldsymbol{G}$ have SzT-type with the same parameter $\alpha$. Then

$$
\mathrm{E}^{3}(A) \ll \mathrm{E}_{3 / 2}^{2}(A) c(A)|A|^{\alpha},
$$




$$
\mathrm{E}(A) \ll c^{1 / 2}(A)|A|^{1+\alpha / 2},
$$

and

$$
\begin{aligned}
& \sum_{x}(A \circ A)(x)(B \circ B)(x)(C \circ C)(x) \\
& \ll(c(A) c(B) c(C))^{1 / 3}(|A||B||C|)^{\alpha / 3} \times \log (\min \{|A|,|B|,|C|\}) .
\end{aligned}
$$

Proof. We prove just (11); estimates (12) and (13) can be established by similar arguments.

Let us arrange the convolutions $(A \circ A)(x)$ in decreasing order: $(A \circ A)\left(x_{1}\right) \geq$ $(A \circ A)\left(x_{2}\right) \geq \cdots$. By assumption $A$ has SzT-type with parameter $\alpha$, which implies that $(A \circ A)\left(x_{j}\right) \ll\left(c(A)|A|^{\alpha} j^{-1}\right)^{1 / 3}$. Choosing the parameter $\Delta^{3 / 2}=$ $c(A)|A|^{\alpha} \mathrm{E}_{3 / 2}^{-1}(A)$ and applying the obtained bound, we get

$$
\mathrm{E}(A)=\sum_{j=1}^{|A-A|}(A \circ A)^{2}\left(x_{j}\right) \leq \Delta^{1 / 2} \mathrm{E}_{3 / 2}(A)+\sum_{j:(A \circ A)\left(x_{j}\right) \geq \Delta}\left(c(A)|A|^{\alpha} j^{-1}\right)^{2 / 3} .
$$

The condition $(A \circ A)\left(x_{j}\right) \geq \Delta$ implies $j^{1 / 3} \ll\left(c(A)|A|^{\alpha}\right)^{1 / 3} \Delta^{-1}$. Thus by our choice of $\Delta$, we have

$$
\mathrm{E}(A) \ll \Delta^{1 / 2} \mathrm{E}_{3 / 2}(A)+c(A)|A|^{\alpha} \Delta^{-1} \ll \mathrm{E}_{3 / 2}^{2 / 3}(A)\left(c(A)|A|^{\alpha}\right)^{1 / 3}
$$

as required.

We need Lemma 7 from [Raz et al. 2015] (see also Lemma 27 from [Schoen and Shkredov 2013]).

Lemma 8. Any set $A \subset \mathbb{R}, \mathbb{R}=(\mathbb{R},+)$, has SzT-type with $\alpha=2$ and $c(A)=|A| d(A)$, where

$$
d(A):=\min _{C \neq \varnothing} \frac{|A C|^{2}}{|A||C|} .
$$

So, any set with small multiplicative doubling or, more precisely, with small quantity (14) has SzT-type, relative to addition, in an effective way. The interested reader can check that the minimum in (14) is actually attained. Careful analysis of our proof shows that we do not need this. Other examples of SzT-type sets can be found in [Shkredov 2015].

Now let us prove a simple result on $d(A)$ that follows from Petridis's Theorem 4.

Lemma 9. Let $A \subseteq \mathbb{R}^{+}$be a set. Then $d(A)=d\left(A^{-1}\right)$, and for any nonempty $C$ we have

$$
d(A A) \leq \frac{|A C|^{4}}{|A A||C|^{3}}, \quad d(A: A) \leq \frac{|A C|^{4}}{|A: A||C|^{3}} .
$$


In particular,

$$
d(A A) \leq \frac{|A|^{2} d^{2}(A)}{|A A||C|}, \quad d(A: A) \leq \frac{|A|^{2} d^{2}(A)}{|A: A||C|},
$$

where $C$ is a set where the minimum in (14) is attained.

Proof. The identity $d(A)=d\left(A^{-1}\right)$ is obvious. Let us prove (16). By Theorem 4 there is $X \subseteq C$ such that $|A A X| \leq R|A X|$, where $R=R_{A}[C]$ is defined by formula (7). We have

$$
d(A A) \leq \frac{|A A X|^{2}}{|A A||X|} \leq R^{2} \frac{|A X|^{2}}{|A A||X|}=\frac{|A X|^{4}}{|A A||X|^{3}} \leq \frac{|A C|^{4}}{|A A||C|^{3}}
$$

and the first bound of (15) is obtained. Similarly, let $Y \subseteq C$ be as given by Theorem 4 and put $R=R_{A}\left[C^{-1}\right]$. Then $|(A: A) Y| \leq R\left|A^{-1} Y\right| \leq R\left|A^{-1} C\right|, R=\left|A Y^{-1}\right| /|Y| \leq$ $\left|A C^{-1}\right| /|C|$, and arguments similar to (17) can be applied.

Finally, we formulate a full version of Theorem 1.

Theorem 10. Let $A, B \subseteq \mathbb{R}$ be sets, and let $\tau>0$ be a real number. Then

$$
|\{x:|A \cap x B| \geq \tau\}| \ll \frac{|A+A||B+B|}{\tau^{2}} .
$$

In particular,

$$
\mathrm{E}^{\times}(A, B) \ll|A+A||B+B| \cdot \log (\min \{|A|,|B|\}) .
$$

\section{Proof of the main results}

Our proof relies on a partial case of Theorem 14 from [Shkredov 2015].

Theorem 11. Suppose $A, A_{*} \subset \mathbb{R}$ have SzT-type with the same parameter $\alpha=2$. Then

(20) $\left|A \pm A_{*}\right| \gg$

$$
\begin{array}{r}
\max \left\{d\left(A_{*}\right)^{-1 / 3} d(A)^{-2 / 9}\left|A_{*}\right|^{8 / 9}|A|^{2 / 3}, d(A)^{-1 / 3} d\left(A_{*}\right)^{-2 / 9}|A|^{8 / 9}\left|A_{*}\right|^{2 / 3},\right. \\
\left.\min \left\{d\left(A_{*}\right)^{-2 / 27} d(A)^{-13 / 27}\left|A_{*}\right|^{14 / 9}, d(A)^{-2 / 27} d\left(A_{*}\right)^{-13 / 27}|A|^{14 / 9}\right\}\right\} \\
\times\left(\log \left(|A|\left|A_{*}\right|\right)\right)^{-2 / 9} .
\end{array}
$$

Now we can prove the main result of the paper.

Theorem 12. Let $A$ be a finite subset of positive reals. Then

$$
|A: A+A| \gg|A|^{3 / 2+1 / 82} \cdot(\log |A|)^{-2 / 41},
$$

and

$$
|A A+A| \gg|A A|^{11 / 41}|A: A|^{-11 / 41}|A|^{3 / 2+1 / 82}(\log |A|)^{-2 / 41} .
$$


Proof. Put $l=\log |A|$. We will assume that $|A: A+A| \ll M|A|^{3 / 2}$ and that $|A A+A| \ll M|A|^{3 / 2}$, where $M$ is a small power of $|A|$, that is, $M=|A|^{\varepsilon}$, and obtain a contradiction. Let us begin with (21) because the proof of the second inequality requires some additional steps.

Recall the arguments from [Balog 2011] or see the proof of Theorem 31 from [Schoen and Shkredov 2013]. Let $l_{i}$ be the line $y=q_{i} x$. Thus, $(x, y) \in l_{i} \cap A^{2}$ if and only if $x \in A_{q}^{\times}$. Let $q_{1}, \ldots, q_{n} \in \Pi \subseteq A$ : $A$ be such that $q_{1}<q_{2}<\cdots<q_{n}$. Here $\Pi$ is a set which can vary, in principle, and at the moment we choose $\Pi$ such that $\left|A_{q_{i}}^{\times}\right| \geq 2^{-1}|A|^{2} /|A: A|$ for all $q_{i} \in \Pi$. Thus, $\sum_{q_{i} \in \Pi}\left|A_{q_{i}}^{\times}\right| \geq \frac{1}{2}|A|^{2}$. We multiply all points of $A^{2}$ lying on the line $l_{i}$ by $\Delta\left(A^{-1}\right)$, so we obtain $\left|A_{q_{i}}^{\times}: A\right|$ points still belonging to the line $l_{i}$, and then we consider the sumset of the resulting set with $l_{i+1} \cap A^{2}$. Clearly, we get $\left|A_{q_{i}}^{\times}: A\right|\left|A_{q_{i+1}}^{\times}\right|$points from the set $(A: A+A)^{2}$ lying between the lines $l_{i}$ and $l_{i+1}$. Put

$$
d(A) \leq \tilde{d}(A):=\min _{i=2, \ldots, n} \frac{\left|A A_{q_{i}}^{\times}\right|^{2}}{|A|\left|A_{q_{i}}^{\times}\right|} .
$$

Therefore, using the definition of $\tilde{d}(A)$, we have

$$
\begin{aligned}
M^{2}|A|^{3} & \gg|A: A+A|^{2} \\
& \geq \sum_{i=1}^{n-1}\left|A_{q_{i}}^{\times}\right|\left|A_{q_{i+1}}^{\times}: A\right| \\
& \geq|A|^{1 / 2} \tilde{d}^{1 / 2}(A) \sum_{i=1}^{n-1}\left|A_{q_{i}}^{\times}\right|\left|A_{q_{i+1}}^{\times}\right|^{1 / 2} \\
& \gg|A|^{3 / 2} \tilde{d}^{1 / 2}(A)|A: A|^{-1 / 2} \sum_{i=1}^{n-1}\left|A_{q_{i}}^{\times}\right| \\
& \gg|A|^{7 / 2} \tilde{d}^{1 / 2}(A)|A: A|^{-1 / 2} .
\end{aligned}
$$

Thus,

$$
d(A) \leq \tilde{d}(A)=\min _{i=2, \ldots, n} \frac{\left|A A_{q_{i}}^{\times}\right|^{2}}{|A|\left|A_{q_{i}}^{\times}\right|} \ll \frac{M^{4}|A: A|}{|A|} .
$$

To estimate $d(A: A)$ and $d(A A)$ we use Lemma 9. In other words, taking our $C=A_{q_{i}}^{\times}$to minimize (25), we get

$$
d(A A) \leq \frac{\left|A A_{q_{i}}^{\times}\right|^{4}}{|A A|\left|A_{q_{i}}^{\times}\right|^{3}}, \quad d(A: A) \leq \frac{\left|A A_{q_{i}}^{\times}\right|^{4}}{|A: A|\left|A_{q_{i}}^{\times}\right|^{3}} \ll \frac{M^{8}|A: A|}{|C|} .
$$


Applying the first inequality of Theorem 11 with $A=A$ and $A_{*}=A: A$, we obtain

$$
\begin{aligned}
M|A|^{3 / 2} & \geq|A: A+A| \\
& \gg|A: A|^{8 / 9}|A|^{2 / 3} d^{-2 / 9}(A)\left(\frac{M^{8}|A: A|}{|C|}\right)^{-1 / 3} l^{-2 / 9} \\
& =|A: A|^{5 / 9}|A|^{2 / 3} d^{-2 / 9}(A)|C|^{1 / 3} M^{-8 / 3} l^{-2 / 9} \\
& \gg|A|^{14 / 9} M^{-32 / 9} l^{-2 / 9}
\end{aligned}
$$

and hence $M \gg l^{-2 / 41}|A|^{1 / 82}$. This implies (21).

It remains to prove (22). In this case we multiply all points of $A^{2}$ lying on the line $l_{i}$ by $\Delta(A)$, so we obtain $\left|A A_{q_{i}}^{\times}\right|$points still belonging to the line $l_{i}$, and then we consider the sumset of the resulting set with $l_{i+1} \cap A^{2}$. Clearly, we obtain $\left|A A_{q_{i}}^{\times}\right|\left|A_{q_{i+1}}^{\times}\right|$points from the set $(A A+A)^{2}$. Thus,

$$
M^{2}|A|^{3} \gg|A A+A|^{2} \geq \sum_{i=1}^{n-1}\left|A_{q_{i}}^{\times}\right|\left|A A_{q_{i+1}}^{\times}\right|,
$$

and we repeat the arguments above. The proof gives us

$$
|A A+A| \gg|A A|^{11 / 41}|A|^{-4 / 41}\left(\mathrm{E}_{3 / 2}^{\times}(A)\right)^{22 / 41} l^{-2 / 41} .
$$

Here we have chosen the set $\Pi$ as $\sum_{q \in \Pi}\left|A_{q}^{\times}\right|^{3 / 2} \gg \mathrm{E}_{3 / 2}^{\times}(A)$ or, in other words, $\left|A_{q}^{\times}\right| \gg\left(\mathrm{E}_{3 / 2}^{\times}(A)\right)^{2}|A|^{-4}$. Using the Hölder inequality, combined with (28), we get

$$
|A A+A| \gg|A A|^{11 / 41}|A: A|^{-11 / 41}|A|^{62 / 41} l^{-2 / 41} .
$$

Remark 13. Using the full power of Theorem 14 from [Shkredov 2015], one can obtain further results connecting $|A A: A|$ and $|A: A A|$ with $|A A+A|$ and $|A: A+A|$ and so on. We do not make such calculations.

The same method allows us to improve the result of Balog concerning the size of $A A+A A$ and $A: A+A: A$.

Theorem 14. Let $A \subset \mathbb{R}$ be a set. Then

$$
|A: A+A: A| \gg|A: A|^{14 / 29}|A|^{30 / 29}(\log |A|)^{-2 / 29},
$$

and

$$
|A A+A A| \gg|A A|^{19 / 29}|A: A|^{-5 / 29}|A|^{30 / 29}(\log |A|)^{-2 / 29} .
$$

Proof. As in the proof of Theorem 12, we define $l_{i}$ to be the line $y=q_{i} x$ and let $q_{1}, \ldots, q_{n} \in \Pi \subseteq A: A$ be such that $q_{1}<q_{2}<\cdots<q_{n}$ and $\left|A_{q_{i}}^{\times}\right| \geq 2^{-1}|A|^{2} /|A: A|$ for any $q_{i} \in \Pi$. Thus, $\sum_{i}\left|A_{q_{i}}^{\times}\right| \geq \frac{1}{2}|A|^{2}$. We multiply all points of $A^{2}$ lying on the line $l_{i}$ by $\Delta\left(A^{-1}\right)$, so we obtain $\left|A_{q_{i}}^{\times}: A\right|$ points still belonging to the line $l_{i}$, and then we consider the sumset of the resulting set with itself. Clearly, we get 
$\left|A_{q_{i}}^{\times}: A\right|\left|A_{q_{i+1}}^{\times}: A\right|$ points from the set $(A: A+A: A)^{2}$ lying between the lines $l_{i}$ and $l_{i+1}$. Therefore, we have

$$
\begin{aligned}
\sigma^{2} & :=|A: A+A: A|^{2} \\
& \geq \sum_{i=1}^{n-1}\left|A_{q_{i}}^{\times}: A\right|\left|A_{q_{i+1}}^{\times}: A\right| \\
& \geq \tilde{d}(A)|A| \sum_{i=1}^{n-1}\left|A_{q_{i}}^{\times}\right|^{1 / 2}\left|A_{q_{i+1}}^{\times}\right|^{1 / 2} \\
& \gg|A|^{3} \tilde{d}(A),
\end{aligned}
$$

where

$$
\tilde{d}(A):=\min _{i=1, \ldots, n} \frac{\left|A_{q_{i}}^{\times}: A\right|^{2}}{|A|\left|A_{q_{i}}^{\times}\right|} .
$$

This gives us $d(A) \leq \tilde{d}(A) \ll \sigma^{2}|A|^{-3}$. Using Theorem 11 with $A=A_{*}=A: A$, we obtain

$$
\begin{aligned}
\sigma & \gg|A: A|^{14 / 9}\left(\frac{\sigma^{4}}{|A|^{4}|A: A||C|}\right)^{-5 / 9} l^{-2 / 9} \\
& \gg|A: A|^{19 / 9}|A|^{20 / 9}|C|^{5 / 9} \sigma^{-20 / 9} l^{-2 / 9} \\
& \gg|A: A|^{14 / 9} \sigma^{-20 / 9}|A|^{10 / 3} l^{-2 / 9} .
\end{aligned}
$$

After some calculations, we get $\sigma \gg|A: A|^{14 / 29}|A|^{30 / 29} l^{-2 / 29}$.

To obtain (30) we use the previous arguments. We have

$$
\begin{aligned}
\sigma^{2} & :=|A A+A A|^{2} \\
& \geq \sum_{i \in \Pi}\left|A A_{q_{i}}^{\times}\right|\left|A A_{q_{i+1}}^{\times}\right| \\
& \geq d(A)|A| \sum_{i \in \Pi}\left|A_{q_{i}}^{\times}\right|^{1 / 2}\left|A_{q_{i+1}}^{\times}\right|^{1 / 2} \\
& \gg d(A)|A||\Pi| \Delta,
\end{aligned}
$$

choosing $\Pi \subseteq A: A$ such that for any $q \in \Pi$ one has $|A|^{2} /|A: A| \ll \Delta \leq\left|A_{q}^{\times}\right|$. Clearly, such a set $\Pi$ exists by simple average arguments. Calculations like those in (33) give us

$$
\sigma \gg|A A|^{14 / 9}\left(\frac{\sigma^{4}}{|A A||\Pi|^{2} \Delta^{3}}\right)^{-5 / 9} l^{-2 / 9} \gg|A A|^{19 / 9}\left(|\Pi| \Delta^{3 / 2}\right)^{10 / 9} \sigma^{-20 / 9} l^{-2 / 9} .
$$

After some computations, we obtain

$$
\sigma \gg|A A|^{19 / 29}|A: A|^{-5 / 29}|A|^{30 / 29} l^{-2 / 29} .
$$


Finally, let us obtain a result on $A A+A$ and $A A+A A$ of another type.

Proposition 15. Let $A \subset \mathbb{R}$ be a set. Then

$$
|A A+A|^{4},|A: A+A|^{4} \gg|A|^{-2}\left(\mathrm{E}_{3 / 2}^{\times}(A)\right)^{2} \mathrm{E}_{3}^{+}(A) \log ^{-3}|A|,
$$

and

$$
|A A+A A|^{2},|A: A+A: A|^{2} \gg \mathrm{E}_{3}^{+}(A) \log ^{-3}|A| .
$$

Moreover,

$$
|A A+A|^{4},|A: A+A|^{4} \gg \frac{|A|^{10}}{|A: A||A-A|^{2}},
$$

and

$$
|A A+A A|^{2},|A: A+A: A|^{2} \gg \frac{|A|^{6}}{|A-A|^{2}} .
$$

Proof. Put $l=\log |A|$. Using Lemma 7, we obtain that for any $A, B$ and $C$

$$
\begin{aligned}
\sum_{x}(A \circ A)(x)(B \circ B)(x) & (C \circ C)(x) \\
& \ll|A||B||C|(d(A) d(B) d(C))^{1 / 3} \log (|A||B||C|) .
\end{aligned}
$$

In the particular case $A=B=C$, the definition of $d(A)$ gives us

$$
\left|A A_{s}^{\times}\right|^{2},\left|A: A_{s}^{\times}\right|^{2} \gg|A|^{-2}\left|A_{s}^{\times}\right| \mathrm{E}_{3}^{+}(A) l^{-1}
$$

for any $s \in A: A$. Using pigeonholing, choose $\Pi \subseteq A: A$ such that $\left|A_{q}^{\times}\right|$differs at most twice from $\Pi$ and such that $\sum_{q \in \Pi}\left|A_{q}^{\times}\right|^{3 / 2} \gg \mathrm{E}_{3 / 2}^{\times}(A) l^{-1}$. Applying (24), (27), (40) and the last bound, we obtain (35). Using (40) one more time and Katz-Koester inclusion [2010], namely,

$$
A A_{s}^{\times} \subseteq A A \cap s A A, \quad A: A_{s}^{\times} \subseteq(A: A) \cap s^{-1}(A: A),
$$

as well as formula (18) of Solymosi's result, we get (36). Another way to prove (36) is just to use formulas (31) and (34), combined with (40).

Inequalities (37) and (38) follow similarly to (35) and (36) from a direct application of Definition 6 and the Hölder inequality. For example, let us show how to get 
the first estimate of (37). Taking $B=-A$ and the parameter $\tau=|A|^{2} /(2|A-A|)$ in Definition 6, we obtain

$$
d(A) \gg \frac{|A|^{3}}{|A-A|^{2}} .
$$

Applying (24) and the lower bound for $d(A)$, we get

$$
\begin{aligned}
|A: A+A|^{2} & \geq \sum_{i=1}^{n-1}\left|A_{q_{i}}^{\times}\right|\left|A_{q_{i+1}}^{\times}: A\right| \\
& \geq|A|^{1 / 2} d^{1 / 2}(A) \sum_{i=1}^{n-1}\left|A_{q_{i}}^{\times}\right|\left|A_{q_{i+1}}^{\times}\right| \\
& \gg|A|^{1 / 2}\left(|A|^{3}|A-A|^{-2}\right)^{1 / 2}\left(|A|^{2} /|A: A|\right)^{1 / 2} \sum_{i=1}^{n-1}\left|A_{q_{i}}^{\times}\right| \\
& \gg|A|^{5 / 2}\left(|A|^{3}|A-A|^{-2}\right)^{1 / 2}\left(|A|^{2} /|A: A|\right)^{1 / 2}
\end{aligned}
$$

as required.

Remark 16. Applying arguments in the proof of (36) as well as formula (12) of Lemma 7, we obtain a similar bound, namely,

$$
\mathrm{E}^{+}(A) \ll|A||A A+A A|
$$

(actually, using methods from [Shkredov 2013a] one can improve the inequality). It is interesting to compare this estimate with Solymosi's upper bound for the multiplicative energy (19). Using formula (11) of Lemma 7, we also have

$$
\left(\mathrm{E}^{+}(A)\right)^{3 / 2} \mathrm{E}_{3 / 2}^{\times}(A) \ll \mathrm{E}_{3 / 2}^{+}(A)|A||A A+A|^{2} .
$$

Combining inequality (36) with some estimates from [Shkredov 2014], we obtain a result in the spirit of [Roche-Newton and Zhelezov 2015].

Corollary 17. Let $A \subset \mathbb{R}$ be a set. Suppose that

$$
|(A+A)(A+A)+(A+A)(A+A)| \ll|A|^{2} \text { and } \mathrm{E}^{+}(A)|A-A| \ll|A|^{4} .
$$

Then

$$
|A-A| \ll|A| \log ^{12 / 7}|A|
$$

The same holds if one replaces addition with subtraction and multiplication with division in the first condition of (42). 
If just the first condition of (42) holds (with plus) then

$$
|A \pm A| \ll|A| \log ^{3}|A|,
$$

and if it holds with minus then

$$
|A-A| \ll|A| \log ^{3}|A|
$$

Again, one can replace multiplication with division in the first condition of (42).

Proof. Let us deal with the situation of the sum and the product. Other cases can be considered similarly. By Theorem 30 from [Shkredov 2014] and our second condition, one has

$$
\mathrm{E}_{3}^{+}(A \pm A) \geq|A|^{45 / 4}|A-A|^{-1 / 2}\left(\mathrm{E}^{+}(A)\right)^{-9 / 4} \gg|A|^{9 / 4}|A-A|^{7 / 4} .
$$

On the other hand, using formula (36) from Proposition 15 and our first condition, we get

$$
|A|^{4} \log ^{3}|A| \gg \mathrm{E}_{3}^{+}(A \pm A) \gg|A|^{9 / 4}|A-A|^{7 / 4}
$$

as required.

Finally, using the additive variant of Katz-Koester inclusion (41) (or see Proposition 29 from [Shkredov 2014]), we obtain

$$
|A|^{3}|A \pm A| \leq \mathrm{E}_{3}^{+}(A+A) \ll|A|^{4} \log ^{3}|A|,
$$

and

$$
|A|^{3}|A-A| \leq \mathrm{E}_{3}^{+}(A-A) \ll|A|^{4} \log ^{3}|A| .
$$

A simpler proof of a stronger result was kindly pointed out to the author by Oliver Roche-Newton. Indeed applying estimate (2) with $A=B=A+A, C=A$ and $D=A+A$, we obtain

$$
\begin{aligned}
|A|^{4} & \gg|(A+A) A+(A+A)(A+A)|^{2} \\
& \gg|(A+A):(A+A)||A||A+A| \\
& \gg|A|^{3}|A+A|,
\end{aligned}
$$

and the result follows. Here we have used the estimate $|(A+A):(A+A)| \geq|A|^{2}$ from [Balog and Roche-Newton 2015]. Applying the well-known Ungar bound $|(A-A):(A-A)| \geq|A|^{2}$ and taking $C=A^{-1}$ and $D=(A+A)^{-1}$, one can replace division with multiplication. 


\section{Acknowledgements}

The author is grateful to the referee for careful reading, to Tomasz Schoen for useful discussions and to Oliver Roche-Newton, who pointed out a short way to obtain a strong form of Corollary 17.

\section{References}

[Balog 2011] A. Balog, "A note on sum-product estimates", Publ. Math. Debrecen 79:3-4 (2011), 283-289. MR 2907965 Zbl 1249.11035

[Balog and Roche-Newton 2015] A. Balog and O. Roche-Newton, "New sum-product estimates for real and complex numbers", Discrete Comput. Geom. 53:4 (2015), 825-846. MR 3341581 Zbl 06450890

[Erdős and Szemerédi 1983] P. Erdős and E. Szemerédi, "On sums and products of integers", pp. 213218 in Studies in pure mathematics: to the memory of Paul Turán, edited by P. Erdôs et al., Birkhäuser, Basel, 1983. MR 86m:11011 Zbl 0526.10011

[Katz and Koester 2010] N. H. Katz and P. Koester, "On additive doubling and energy", SIAM J. Discrete Math. 24:4 (2010), 1684-1693. MR 2012d:11020 Zbl 1226.05247

[Konyagin and Rudnev 2013] S. V. Konyagin and M. Rudnev, "On new sum-product-type estimates", SIAM J. Discrete Math. 27:2 (2013), 973-990. MR 3056747 Zbl 1272.68328

[Li 2011] L. Li, "On a theorem of Schoen and Shkredov on sumsets of convex sets", preprint, 2011. arXiv 1108.4382

[Li and Roche-Newton 2012] L. Li and O. Roche-Newton, "Convexity and a sum-product type estimate”, Acta Arith. 156:3 (2012), 247-255. MR 2999071 Zbl 1279.11013

[Murphy et al. 2015] B. Murphy, O. Roche-Newton, and I. D. Shkredov, "Variations on the sumproduct problem”, SIAM J. Discrete Math. 29:1 (2015), 514-540. MR 3323540 Zbl 06437890

[Petridis 2012] G. Petridis, "New proofs of Plünnecke-type estimates for product sets in groups", Combinatorica 32:6 (2012), 721-733. MR 3063158 Zbl 1291.11127

[Raz et al. 2015] O. E. Raz, O. Roche-Newton, and M. Sharir, "Sets with few distinct distances do not have heavy lines", Discrete Math. 338:8 (2015), 1484-1492. MR 3336119 Zbl 1310.05039

[Roche-Newton and Zhelezov 2015] O. Roche-Newton and D. Zhelezov, "A bound on the multiplicative energy of a sum set and extremal sum-products problems", Mosc. J. Comb. Number Theory 5:1-2 (2015). arXiv 1410.1156

[Schoen 2014] T. Schoen, "On convolutions of convex sets and related problems", Canad. Math. Bull. 57:4 (2014), 877-883. MR 3270808 Zbl 06377673

[Schoen and Shkredov 2011] T. Schoen and I. D. Shkredov, "On sumsets of convex sets", Combin. Probab. Comput. 20:5 (2011), 793-798. MR 2012g:11021 Zbl 1306.11013

[Schoen and Shkredov 2013] T. Schoen and I. D. Shkredov, "Higher moments of convolutions", J. Number Theory 133:5 (2013), 1693-1737. MR 3007128 Zbl 1300.11018

[Shkredov 2013a] I. D. Shkredov, "Some new inequalities in additive combinatorics", Mosc. J. Comb. Number Theory 3:3-4 (2013), 189-239. MR 3284125 Zbl 06367620

[Shkredov 2013b] I. D. Shkredov, "Несколько новых результатов о старших энергиях", Tr. Mosk. Mat. Obs. 74:1 (2013), 35-73. Translated as "Some new results on higher energies" in Trans. Mosc. Math. Soc. 74 (2013), 31-63. MR 3235789 Zbl 06371555 
[Shkredov 2014] I. D. Shkredov, "Energies and structure of additive sets", Electron. J. Combin. 21:3 (2014), Paper \#P3.44. MR 3262281 Zbl 1301.11010

[Shkredov 2015] I. D. Shkredov, "O суммах множеств Семереди-Троттера", Tr. Mat. Inst. Steklova 289 (2015), 318-327. Translated as "On sums of Szemerédi-Trotter sets" in Proc. Steklov Inst. Math. 289:1 (2015), 300-309.

[Solymosi 2009] J. Solymosi, "Sumas contra productos", Gac. R. Soc. Mat. Esp. 12:4 (2009), 707719. MR 2589308 Zbl 1284.11019

[Tao and Vu 2006] T. Tao and V. Vu, Additive combinatorics, Cambridge Studies in Advanced Mathematics 105, Cambridge University Press, 2006. MR 2008a:11002 Zbl 1127.11002

Received February 2, 2015. Revised May 23, 2015.

ILYA D. SHKREDOV

STEKLOV MATHEMATICAL InSTITUTE

UL. GUBKINA, 8

Moscow

119991

RUSSIA

and

IITP RAS

BOLSHOY KARETNY PER. 19

Moscow

127994

RUSSIA

ilya.shkredov@gmail.com 


\title{
PACIFIC JOURNAL OF MATHEMATICS
}

\author{
msp.org/pjm
}

Founded in 1951 by E. F. Beckenbach (1906-1982) and F. Wolf (1904-1989)

\section{EDITORS}

Don Blasius (Managing Editor)

Department of Mathematics

University of California

Los Angeles, CA 90095-1555

blasius@math.ucla.edu

\author{
Paul Balmer \\ Department of Mathematics \\ University of California \\ Los Angeles, CA 90095-1555 \\ balmer@math.ucla.edu \\ Robert Finn \\ Department of Mathematics \\ Stanford University \\ Stanford, CA 94305-2125 \\ finn@math.stanford.edu \\ Sorin Popa \\ Department of Mathematics \\ University of California \\ Los Angeles, CA 90095-1555 \\ popa@math.ucla.edu
}

\author{
Vyjayanthi Chari \\ Department of Mathematics \\ University of California \\ Riverside, CA 92521-0135 \\ chari@math.ucr.edu \\ Kefeng Liu \\ Department of Mathematics \\ University of California \\ Los Angeles, CA 90095-1555 \\ liu@math.ucla.edu \\ Jie Qing \\ Department of Mathematics \\ University of California \\ Santa Cruz, CA 95064 \\ qing@ cats.ucsc.edu
}

\section{PRODUCTION}

Silvio Levy, Scientific Editor, production@msp.org

\section{SUPPORTING INSTITUTIONS}

ACADEMIA SINICA, TAIPEI

CALIFORNIA INST. OF TECHNOLOGY

INST. DE MATEMÁTICA PURA E APLICADA

KEIO UNIVERSITY

MATH. SCIENCES RESEARCH INSTITUTE

NEW MEXICO STATE UNIV.

OREGON STATE UNIV.

\author{
STANFORD UNIVERSITY \\ UNIV. OF BRITISH COLUMBIA \\ UNIV. OF CALIFORNIA, BERKELEY \\ UNIV. OF CALIFORNIA, DAVIS \\ UNIV. OF CALIFORNIA, LOS ANGELES \\ UNIV. OF CALIFORNIA, RIVERSIDE \\ UNIV. OF CALIFORNIA, SAN DIEGO \\ UNIV. OF CALIF., SANTA BARBARA
}

\author{
Daryl Cooper \\ Department of Mathematics \\ University of California \\ Santa Barbara, CA 93106-3080 \\ cooper@math.ucsb.edu \\ Jiang-Hua Lu \\ Department of Mathematics \\ The University of Hong Kong \\ Pokfulam Rd., Hong Kong \\ jhlu@maths.hku.hk \\ Paul Yang \\ Department of Mathematics \\ Princeton University \\ Princeton NJ 08544-1000 \\ yang@math.princeton.edu
}

These supporting institutions contribute to the cost of publication of this Journal, but they are not owners or publishers and have no responsibility for its contents or policies.

See inside back cover or msp.org/pjm for submission instructions.

The subscription price for 2016 is US $\$ 440 /$ year for the electronic version, and $\$ 600 /$ year for print and electronic.

Subscriptions, requests for back issues and changes of subscribers address should be sent to Pacific Journal of Mathematics, P.O. Box 4163, Berkeley, CA 94704-0163, U.S.A. The Pacific Journal of Mathematics is indexed by Mathematical Reviews, Zentralblatt MATH, PASCAL CNRS Index, Referativnyi Zhurnal, Current Mathematical Publications and Web of Knowledge (Science Citation Index).

The Pacific Journal of Mathematics (ISSN 0030-8730) at the University of California, c/o Department of Mathematics, 798 Evans Hall \#3840, Berkeley, CA 94720-3840, is published twelve times a year. Periodical rate postage paid at Berkeley, CA 94704, and additional mailing offices. POSTMASTER: send address changes to Pacific Journal of Mathematics, P.O. Box 4163, Berkeley, CA 94704-0163.

PJM peer review and production are managed by EditFLOW ${ }^{\circledR}$ from Mathematical Sciences Publishers.

\section{PUBLISHED BY}

\section{mathematical sciences publishers \\ nonprofit scientific publishing}

http://msp.org/

(C) 2016 Mathematical Sciences Publishers 


\section{PACIFIC JOURNAL OF MATHEMATICS}

Volume $280 \quad$ No. $1 \quad$ January 2016

Stable capillary hypersurfaces in a wedge

JAIGYOUNG CHOE and MIYUKI KoISO

The Chern-Simons invariants for the double of a compression body

DAVID L. DUNCAN

Compactness and the Palais-Smale property for critical Kirchhoff equations in

closed manifolds

EMMANUEL HeBEy

On the equivalence of the definitions of volume of representations

SUNGWOON KIM

Strongly positive representations of even GSpin groups

YEANSU KIM

An Orlik-Raymond type classification of simply connected 6-dimensional torus manifolds with vanishing odd-degree cohomology

\section{SHINTARÔ KUROKI}

Solutions with large number of peaks for the supercritical Hénon equation

Zhongyuan LiU and ShuangJie Peng

Effective divisors on the projective line having small diagonals and small heights and 141 their application to adelic dynamics

YÛSUKE OKUYAMA

Computing higher Frobenius-Schur indicators in fusion categories constructed from inclusions of finite groups

\section{Peter Schauenburg}

Chordal generators and the hydrodynamic normalization for the unit ball

SEBASTIAN SCHLEISSINGER

On a question of A. Balog

ILYA D. SHKREDOV

Uniqueness result on nonnegative solutions of a large class of differential inequalities 241 on Riemannian manifolds

YUHUA SUN

Correction to "Closed orbits of a charge in a weakly exact magnetic field"

WILL J. MERRY 